\title{
LAS METODOLOGÍAS DE AGENDA EN EL ESTUDIO DE LA OPINIÓN PÚBLICA COMO BASE DE LA COMUNICACIÓN POLÍTICA
}

Fermín Bouza

Universidad Complutense de Madrid bouza@ccinf.ucm.es

Material original autorizado para su primera publicación en la revista académica REDMARKA Revista Digital de Marketing Aplicado

https://doi.org/10.17979/redma.2012.01.08.4731

Recibido: 15 Abril 2012

Aceptado 24 Mayo 2012

\section{RESUMEN}

El estudio de las Agendas a través de la pregunta de agenda en diversas encuestas (es la pregunta sobre cuál el problema más importante del país y/o personal) es una fuente rigurosa de acceso a la Opinión Pública y de preparación de la Comunicación Política, Empresarial,etc. En este artículo se muestra lo esencial de esa metodología y de su eficacia descriptiva. La metodología de agenda es el fundamento de diversas teorías, en particular la Teoría de la Agenda Setting, una de las referencias centrales en la investigación de los efectos de los medios y del análisis de la Agenda Mediática. En esa línea de investigación se inserta este artículo.

Palabras clave: Agenda Setting, Opinión Pública, Comunicación Política.

\section{ABSTRACT}

AGENDA METHODOLOGIES IN THE STUDY OF PUBLIC OPINION AS THE BASIS OF THE POLITICAL COMMUNICATION

${ }^{1}$ Catedrático de Sociología -Opinión Pública- en el Departamento de Sociología VI, Opinión Pública y Cultura de Masas, en la Facultad de Ciencias de la Información de la UCM. Una parte de sus textos figuran en su página web en el departamento citado de la Facultad de Ciencias de la Información de la Universidad Complutense de Madrid. Algunas de sus publicaciones sobre el tema se citan en este artículo 
The study of the Agendas through the agenda question in diverse surveys (it is the question on which the most important problem in the country and/o personal/private) it is a rigorous source of access to the Public Opinion and of preparation of the Political or Managerial Communication, etc. In this article and sample the essential of that methodology and of their descriptive effectiveness. The agenda methodology is the foundation of diverse theories, in particular the Theory of the Agenda Setting, one of the central references in the investigation of the media effects and of the analysis of the Media Agenda. In that investigation line this article is inserted.

Key Words: Agenda Setting, Public Opinion, Political Communication. 


\section{Introducción}

La Comunicación Política fundada en datos se aleja de cualquier construcción comunicativa sobre la base de la intuición o el buen saber de la costumbre. Para elaborar estrategias de comunicación el punto de partida son los datos cualitativos y cuantitativos que nos permiten hablar del mundo con cierto rigor, a los que añadimos, ahora sí, nuestro buen hacer, nuestra costumbre, nuestra creatividad, y cuantas virtudes de esta clase podamos poseer. Esto es una afirmación de principios sobre lo que debe ser una buena comunicación, ya sea política o de cualquier otra clase. A veces los datos vienen ya con el problema, y no es necesario abrir una investigación o una pesquisa especial, pero otras veces desconocemos casi todo de lo que ocurre alrededor de la comunicación que pretendemos elaborar. En el caso de la comunicación político-electoral esto último es lo más frecuente: tenemos datos irregulares de encuestas mal conocidas, apenas sus cifras marginales, o bien datos de prensa, sesgados por principio, o inteligentes intuiciones personales: un magma desordenado y $\sin$ mucha consistencia racional y/o empírica sobre el que podemos cometer todos los errores del mundo al tratar de analizar y comunicar algo.

En estas líneas voy a tratar de explicar por qué y cómo debemos trabajar con las agendas, pero antes definiré qué son esas agendas, muy mal conocidas, incluso por los especialistas, y voy a poner algunos ejemplos. Será un pequeño texto monográfico sobre metodología de agendas, tanto en su aspecto cuantitativo (uso de porcentajes y correlaciones, sobre todo), como cualitativo: metodología de análisis de framing con focus groups o grupos de discusión, sobre todo grupos-panel contrastados con grupos de control, que es la metodología que el grupo Agenda y Voto ha elaborado y usa habitualmente. Se trata de un nuevo instrumento metodológico construido a partir del estudio de la relación medios/voto, de la profundización en los diversos niveles de la teoría de la Agenda-Setting, y de la lectura de los clásicos del análisis de frames, como Entman o Goffman. Las nuevas teorizaciones de los sociólogos sobre los medios (Hallin y Mancini, entre otros) también estuvieron presentes en los giros mediáticos que fuimos dando a nuestra investigación sobre voto (voto y racionalidad) desde el año 2000 en que la comenzamos. Cuando hablo en 
plural me refiero, sobre todo, a la parte madrileña del grupo Agenda y Voto (Juan Jesús González, Raquel Rodríguez, Palmira Chavero, Antón Rodríguez Castromil y yo mismo). Algunos de nosotros, como J.J. González y yo, Fermín Bouza, habíamos trabajado en el área demoscópica y/o comunicacional de algún partido y/o sindicato, o habíamos asesorado a candidatos de partido. Desde esa base abordamos nuestras investigaciones posteriores, entre las que están las relativas a los medios y las agendas.

En el libro reciente de González y Bouza (2009), Las razones del voto en la España democrática (1977-2008), se tocan ya todos estos temas y allí se pueden ampliar muchas de estas cosas a través de diversos capítulos (en el caso de la metodología de agenda hay un anexo especial al final del libro) y de una bibliografía breve pero necesaria. Ahí remito a los interesados en seguir trabajando en estos métodos, enfoques y preocupaciones.

\section{Historia de la Comunicación Política. Origen e inserción científica.}

No hace mucho, en la revista Telos que editó un monográfico sobre Comunicación Política, escribí un breve texto (Bouza, F, 2008) al que remito a la gente interesada y que ahora resumo muy brevemente. Los hechos que trato de subrayar pueden situar con rigor a la ciencia social llamada Comunicación Política, que es también (como toda ciencia) una práctica peculiar que puede tener como instrumento al llamado Marketing Político, y desarrollarse, por tanto, en el área electoral e incluso en la empresarial y comercial. En este sentido, la Comunicación Política nace vinculada a largas y anteriores investigaciones sobre los medios y el voto, las más famosas de las cuales proceden del llamado Grupo de Columbia (Columbia University's Bureau of Applied Social Research) $)^{2}$.

\footnotetext{
${ }^{2}$ Dos citas directas del artículo citado para fijar el origen del concepto y la tradición en la que se inserta:

a).-El especialista Steven Chaffe (Chaffee, S.H. 2001: «Studying the New Communication of Politics», Political Communication, 18, pág. 237) atribuye a Harold Laswell el inicio del estudio de la Comunicación Política (Propaganda technique in the world war, 1927) y del uso del concepto en su sentido académico (Political communication: The public language of political elites in India and the United States, 1969, junto con S. K. Arora).
} 
Se trata de la aparición normal de una ciencia: una tradición y un más o menos claro punto de origen. Pero la tradición de inserción pesa mucho, y esa tradición exige una metodología científica tanto para el trabajo académico de investigación (relación medios/política, esencialmente) como para el trabajo aplicado (Comunicación Política óptima de organizaciones y partidos políticos para optimizar, a su vez, ventas, difusión o, en el caso político estricto, votos).

$Y$ es en este sentido en el que a mí me gusta, como científico, hablar de "Comunicación Política fundada en datos", sin que estoy excluya la creatividad o la imaginación: hay sitio para todo en el campo comunicacional, y con frecuencia los partidos necesitan más creatividad e imaginación que ninguna otra cosa.

\section{Cambios estructurales y nuevos partidos políticos}

Los nuevos partidos políticos, un producto, sobre todo, de la posguerra mundial (segunda guerra), se diferencian de los viejos partidos políticos en que no son ya partidos de masas, como se decía, partidos casi militarizados, convertidos en auténticas organizaciones comunitarias, en el sentido de Tönnies, unidas por vínculos de afecto y emoción, grupos primarios de gran tamaño que eran parte sustancial de la vida de sus militantes y seguidores. Los viejos partidos eran parte central del paisaje decimonónico y finisecular, y prolongaron su organización y eficacia hasta esa frontera de toda clase que fue la segunda guerra mundial. Aquellos partidos eran auténticos partidos de clase, y defendían duramente los intereses que decían representar. Representaban bien lo que fue aquella sociedad finisecular hasta los años cincuenta del siglo $X X$, en el supuesto de que la burguesía y el proletariado eran las grandes clases protagonistas de la historia en aquel momento, con una aristocracia y un campesinado fuera del escenario central que habían ocupado durante tantos siglos. Pero esa sociedad dicotomizada no va a durar mucho, por la propia

b).-Cuando en el año 1975 el ya citado Steven Chaffee, un significado estudioso de los socialización política a través de los medios, publica su reading «Political communication: Issues and strategies for research» (Chaffee, S.H., ed. (1975). Political Communication: Issues and Strategies for Research. Sage Annual Reviews of Communication, Volume IV. Beverly Hills, Califomia, Sage Publications, 1975) éste es recibido, en cierto modo, como una continuación de los estudios de Paul Lazarsfeld y colegas del Grupo de Columbia. 
dinámica del capitalismo emergente, que creará nuevas clases intermedias (lo que se llamarán clases medias, y que teorizará, entre otros, el gran sociólogo americano Wright Mills, siguiendo la huella de Marx y Weber, sobre todo, y que en la actualidad sigue haciendo y ampliando, entre otros, el sociólogo Erik Olin Wright, muy traducido y estudiado en España). La fractura de las viejas clases se produce sobre todo a lo largo del siglo XX en los países avanzados y semiavanzados, y es un proceso en constante expansión que llega poco a poco a todos los extremos del mundo que van entrando en procesos de modernización. La consecuencia de todo ello la resumimos en el libro nuestro ${ }^{3}$ (González y Bouza, 2009): ¿Qué son entonces los nuevos partidos políticos?. Podríamos definirlos como partidos de gestión que se constituyen en oficinas públicas de oferta de ideas para obtener el apoyo democrático suficiente para gobernar. Son partidos que con frecuencia decepcionan a la gente por una razón sencilla: no siempre defienden con fuerza lo que son sus principios básicos, y ello por una razón que les dio vida y sentido: son partidos de paz, democráticos, nacieron para ganar elecciones y convencer, no para ganar batallas en la calle o cosa similar, y no pueden ni deben abrumar a sus adversarios con el $100 \%$ de sus principios: nacieron para negociar y avanzar a través de consensos complejos. Son partidos aburridos si los comparamos con los viejos partidos, su pretensión no es ganar la calle, apenas usarla a veces para manifestarse: su pretensión es ganar votaciones. Se acabaron las meriendas colectivas (familia incluida) de los viejos partidos, se acabaron las manifestaciones uniformadas, el paso militar y las canciones de guerra. Es otro mundo, sin duda más aburrido y pacífico. Lo más divertido es la comunicación partidaria, el modo de hablarle a los votantes, simpatizantes, militantes. E modo de hablarle a todo el mundo, votante o no. Es el mundo partidario de la Comunicación Política. También de la imagen del candidato. Un mundo más espectacular, en este sentido, una auténtica Sociedad del Espectáculo, término

\footnotetext{
${ }^{3}$ Libro citado en nota 1, p. 206: ¿Qué cambios espaciotemporales obligan a modificar nuestra mirada sobre el mundo? Sustancialmente, dos cosas han cambiado a lo largo del siglo XX: en primer lugar, la estructura social de los países avanzados y semiavanzados, con la fragmentación hasta el infinito de las viejas clases sociales y, en segundo lugar, la estructura mediática de esos mismos países, que ha conocido el ascenso y la multiplicación de la televisión y, recientemente, de Internet. Un nuevo mundo mediático y fragmentado para el que ya no sirven los viejos análisis sobre una estructura social estática ni la consideración de los partidos políticos como único y principal sujeto emisor de política y narraciones políticas.
} 
expresivo que acuñó el situacionista Guy Debord (1967), un recuerdo de las también espectaculares y vibrantes ideologías del 68, con su pretensión revolucionaria de cambiar el mundo y con su fuerte carga fideísta y voluntarista. La gran teoría posterior sobre el espectáculo político, muy vinculada al marketing, nace o renace, según y cómo, al albur de la reflexión de Debord, bien para condenar el espectáculo bien para apropiarse de él desde las ideologías y los gabinetes de comunicación.

\section{La nueva retórica mediática y la Comunicación Política: Formateo del discurso político y Factores de noticia}

La Comunicación Política como ciencia de los efectos de los medios (en particular de la televisión) nace cuando la televisión aparece en los EEUU y algunos estudiosos lo entienden, en parte, como una amenaza sobre la política democrática, dado el inmenso poder que parece desplegar. Cito un párrafo de Daniel Bell (1969/1960) que me gusta repetir para mostrar esa potencia en la cabeza de los estudiosos de aquel momento:

La sociedad que carece de instituciones nacionales bien definidas y de clases nacionales conscientes de ser tales, queda amalgamada mediante los medios de comunicación de masas. En la medida en que es posible establecer la fecha de una revolución social, la noche del 7 de marzo de 1955 podría, quizá, tomarse como piedra miliar: aquella noche un americano de cada dos estaba mirando a Mary Martin que se exhibía en "Peter Pan" ante las cámaras de televisión. Jamás hasta entonces, en el curso de la historia, un individuo había sido visto y oído por tantas personas al mismo tiempo. Esto era lo que Adam Smith había llamado la Gran Sociedad, pero mucho más "grande" de lo que él hubieras podido imaginar nunca.

Es decir, para Bell la televisión asigna a la sociedad americana el carácter de nación (en el mismo texto habla sobre ello muy directamente), nada menos, y con este ejemplo de Bell podemos ya imaginar el profundo miedo que todos los intelectuales democráticamente sensibles podrían mostrar ante un demoniaco aparato que es capaz hasta de construir naciones. 
Va a ser una realidad el nuevo ritmo temporal (timing) de la televisión, determinado por la carestía del tiempo en el nuevo medio, que se impone pronto como el más seguido y deseado, por la sobreabundancia de la oferta publicitaria peleando por ese espacio temporal, por los ciudadanos en general $y$, dentro de ellos, por los políticos, que ven en ese nuevo foco narrativo la panacea comunicacional. Pero el precio es la adaptación al medio, que es lo que siempre caracterizó a los sobrevivientes. Y dicho y hecho, la política ajustó su discurso a la brevedad y el colorido que la televisión exigía. Cuatro factores de noticia ${ }^{4}$ van a dominar la narración televisiva y la de los medios en general: la personalización, la negatividad, la sorpresa y la tematización. Y así se irá construyendo lo que se llamó "la democracia centrada en los medios" (David L. Swanson ${ }^{5}$ ), que será la que a su vez de formas a la Comunicación Política como ciencia y como práctica. El uso de los factores de noticia incrementa la posibilidad de que algo se convierta en noticia ${ }^{6}$. La personalización es lo que su nombre indica: la centralización en una persona de una idea, ya sea política o de cualquier otra índole-. Es exactamente lo que ocurre cuando se sustituye una corriente de opinión por un líder, un partido por una sola figura política, sinécdoque pars pro toto que funda el reduccionismo de la idea a su soporte y los procesos de liderazgo que son centrales en toda política contemporánea, que es una política, como hemos visto, de gestión, no de masas, de ideas propuestas, no de debates.

La negatividad es obvia: cuanto peor, mejor o, como dicen que dicen (?) en las facultades de periodismo, "las buenas noticias no son noticia". Quizá no lo

\footnotetext{
${ }^{4}$ Puede verse la historia de estos factores en las notas de mi artículo: Bouza, F. (2004). "The impact Area of Political Communication: Citizenship Faced with Public Discourse", International Review of Sociology - Revue Internationale de Sociologie,Vol. 14, no 2, p. 245- 259. Hay una versión similar en castellano en http://www.ucm.es/info/socvi/BOUZA/NUEVA1/Textos/impactesp.pdf También puede verse la referencia en Donsbach, W. (1995). "Contenidos, utilización y efectos de la Comunicación Política”, en en Muñoz-Alonso, A., y Rospir, J.L. (eds), Comunicación Política, Madrid, Editorial Universitaria, pp. 41-67-

${ }^{5}$ Swanson, D. L.( 1995). "El campo de la Comunicación Política. La democracia centrada en los Medios",en Muñoz-Alonso, A., y Rospir, J.L. (eds), Comunicación Política, Madrid, Editorial Universitaria, pp. 3-24

${ }^{6}$ Sobre los factores de noticia y los procesos de telenovelización de la política puede verse mi artículo sobre el tema, en donde sitúo la cuestión en los procesos de transición económica y política de España: "La telenovelización de la política (del nacionalcatolicismo al mirón comunitario)". Lo que hacen los sociólogos, libro-homenaje a Carlos Moya, CIS, Madrid, 2007, pp.363-373
} 
digan, o al menos yo no lo he oído nunca en mis largos años entre periodistas, pero de hecho es así. En la narración partidaria esto se traduce en un modo de plantear la política en los términos en que el adversario sea el portador perpetuo de malas noticias. Todo lo malo vende, esa sería la filosofía televisiva de fondo, o mediática en general. $\mathrm{O}$, más matizadamente: lo malo vende mejor que lo bueno. Esto obliga a los partidos a negativizar su relato, y así a estas alturas de este pequeño artículo ya lo tenemos personalizado y negativizado. Sigamos disponiendo lugares retóricos de argumentación.

Si ahora añadimos la sorpresa como nuevo factor de noticia, entonces estamos obligados a inventarnos el mundo cada día, algo a lo que la política actual está muy acostumbrada. La política actual tiene algo de género literario- $Y$ así es: todos los días hay alguna novedad y todos los días tratan de llamar nuestra atención con alguna microsorpresa, cuando no con una gran sorpresa, aunque estas últimas suelen guardarse para las campañas electorales. Ya tenemos la narración personalizada, negativizada y sorprendente. Pero aún falto algo.

El último factor citado es la tematización, y alude al ajuste entre el tema tocado y el medio en el que se pretende que aparezca. Se supone que todo medio es "temático" (tiene temas preferentes). Pues bien, cuanto más nos acerquemos a esos temas más probabilidades de que el medio tenga en cuenta nuestro intento de comunicar. Bien. Ya tenemos un discurso personalizado, negativo, sorprendente y temático. Se supone que es el discurso político ideal. Habrá que matizar todo esto con la experiencia y la crítica.

\section{La metodología de Agenda en la preparación de la Campaña Electoral}

Hay varios momentos en los que el uso de las Agendas es fundamental para perfilar diversos eventos de campaña. Pero, ¿qué son las agendas?. En este punto voy a sintetizar (con alguna variación enriquecedora que amplía y profundiza el texto, y también con alguna corrección), en nota al pie (nota 10), algunos párrafos de nuestro trabajo ${ }^{7}$. Es preciso valorar algunas cuestiones de método que hacen de la pregunta de agenda (el problema más importante) una

\footnotetext{
${ }^{7}$ González y Bouza, ob.cit, pp.208-218 (ver ANEXO)
} 
pregunta muy significativa al ser una pregunta abierta (y codificada de acuerdo a criterios aún no internacionalmente homogeneizados) que permite amplios movimientos cognitivos y gran flexibilidad en la contestación, por tanto. En la versión del CIS se dan tres oportunidades de respuesta, entre otras opciones (también se da la versión original de Gallup, que se obtiene en todo caso con una opción simple en el programa estadístico).

El control de la evolución de las agendas (mejor si se hace introduciendo los resultados en un buen programa estadístico, o fusionando los archivos del CIS) nos permitirá saber exactamente la relación del electorado con los temas, y nos permitirá diseñar la Agenda Partidaria de Campaña de tal manera que mantenga alguna coherencia suficiente con la Agenda Publica y con la Agenda Personal. Es una forma precisa de acceder a una Opinión Pública muy sistematizada, de largas series estadísticas, y de carácter internacional. EI MIP (Most Important Problem) es una pregunta que viene de principios del siglo pasado (Gallup y otros), que ha sido realizada infinidad de veces, que ha sido, por supuesto, cuestionada (es muy interesante la literatura científica de su cuestionamiento ${ }^{8}$ ), pero que sigue siendo una fuente central de conocimiento de la opinión pública, sobre todo en el área política, pero no sólo (podemos personalizarla para contextos específicos).

\section{REFERENCIAS}

González, JJ., y Bouza, F. (2009). Las razones del voto en la España democrática (1977-2008). Madrid: Los libros de la catarata.

Bouza, F.(2008). "Una reflexión metodológica sobre la investigación en Comunicación Política", Telos, Enero-Marzo 2008, № 74, Madrid: http://www.campusred.net/TELOS/articulocuaderno.asp?idarticulo=5\&rev $=74$

\footnotetext{
${ }^{8}$ Wlezien, Christopher: "On the salience of political issues: The problem with 'most important problem", Electoral Studies 24 (2005), PP.555-579. También: Young Min, Salma I. Ghanem, and Dixie Evatt: Using a split-ballot survey to explore the robustness of the 'mip' question in agenda-setting research: a methodological study, International Journal of Public Opinion Research Vol. 19, № 2, pp. 221-236
} 
Debord, G (1967). La sociètè du spectacle, Champ Libre, París. Texto íntegro en castellano: http://sindominio.net/ash/espect.htm

Bell, D. (1969/1960). "Modernity and Mass Society. On the Varieties of Cultural Experience", Studies in Public Communication, 4. Por la traducción española en AC editor ("Modernidad y sociedad de masas. Variedad de la experiencia cultural", en La industria de la cultura), Madrid, 1969.

\section{ANEXO}

Hasta hace poco la palabra "agenda" se refería en exclusiva a un pequeño cuaderno en el que apuntábamos los números de teléfono. A veces era algo más: un cuaderno en el que íbamos apuntando tareas y compromisos, y en ese caso la agenda llevaba días, meses y fechas. Era un cuaderno de papel que fue siendo sustituido por unos complicados artefactos llamados agendas electrónicas. . Un tiempo después las agendas para apuntar cosas diversas se fueron incorporando a los teléfonos móviles y a otros artilugios de las nuevas tecnologías. Algún día impreciso dentro de ese proceso se recuperó y se fue imponiendo un significado apenas usado entre nosotros: la agenda es el conjunto de cosas que uno debe hacer o que uno debe tener en cuenta de alguna manera, y a esa agenda, que podía ser sólo un artefacto mental, se añadía generalmente una prioridad temática o una jerarquía, unas veces en función de la proximidad o urgencia y otras en función de su relevancia u otros criterios. Todos teníamos una agenda aunque no tuviéramos ningún cuaderno o ningún artefacto pre o neotecnológico para memorizarla. Entonces ya todo el mundo comenzó a incorporar este significado ("tengo una agenda muy apretada", "ando mal de agenda", etc.). Ése era el significado más usado en el mundo angloamericano, aunque no era ajeno al significado en la lengua castellana, que había sustituido el concepto más abstracto por el formato material de ese concepto, los cuadernos o las agendas electrónicas. Y una vez aclarado esto, que aún podría confundir a algunas personas, podemos ya hablar de las agendas que van elaborando los ciudadanos, los medios de comunicación, los políticos, las instituciones y tantos otros sujetos de la da social. En el campo de los medios y del voto, fue una teoría llamada Agenda- 
Setting (1972) la que comenzó a dar particular relevancia al estudio de las agendas, como iremos viendo. Siguiendo la terminología de esa teoría, con algunos añadidos míos para ajustarla al caso español y a otras cuestiones, vamos a ir mostrando la probable incidencia de las agendas (sobre todo las agendas pública, personal, mediática y política o institucional) en la conducta electoral de los ciudadanos, porque hay una significativa ausencia en el análisis del voto en España: el análisis de los medios de comunicación y su compleja influencia en la racionalidad electoral. En el análisis estrictamente electoral o demoscópico de tipo sociológico o politológico apenas había algún trabajo aislado o alguna difusa referencia. Añadido a esto, la ausencia de un análisis del voto en función de las diversas agendas, en este caso de la agenda mediática, que es el conjunto de propuestas temáticas de los medios de comunicación a la opinión pública y, por tanto, al conjunto de la ciudadanía. Estas propuestas de los medios se pueden contabilizar de diversas maneras, y nosotros (Grupo de Investigación Agenda y Voto) lo hacemos contando las noticias de portada (temas) y las de los editoriales. Pero puede hacerse más 0 menos completa esta contabilidad, según metodologías. En los barómetros mensuales del CIS la agenda pública y la agenda personal tienen la forma de dos preguntas encabalgadas. Agenda pública: ¿Cuál es, a su juicio, el principal problema que existe actualmente en España? ¿Y el segundo? ¿Y el tercero?. Agenda personal: ¿Y cuál es el problema que a usted, personalmente, le afecta más? ¿Y el segundo? ¿Y el tercero. Enseguida veremos con ejemplos estos conceptos que nos van a llevar a entender una parte importante de la razón del voto. Para empezar podemos quedarnos con la referencia de la figura, que alude a la relación entre estas cuatro agendas citadas arriba: es una relación poco o muy poco interactiva, según y cómo, como iremos viendo. Estas son las cuatro Agendas de referencia y sus interacciones más significativas: 


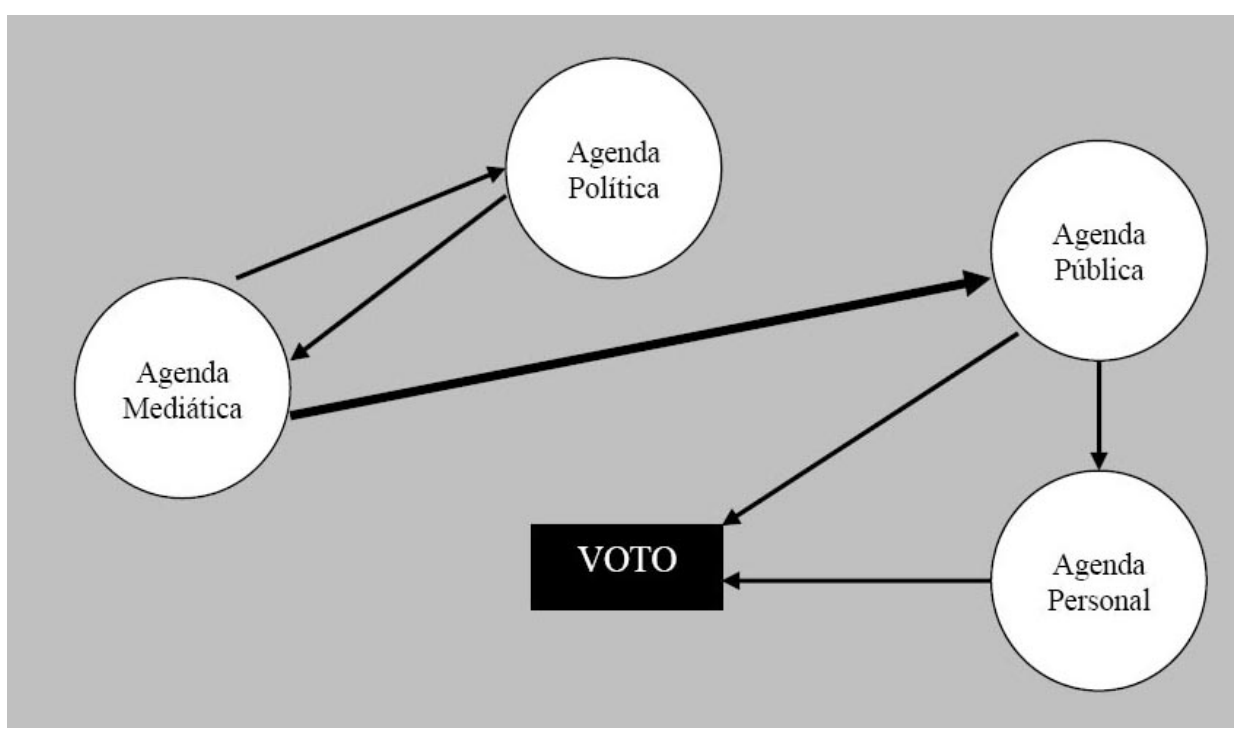

La agenda mediática incluye los temas que nos proponen los medios como prioritarios y que los medios nos transmiten o transfieren a los ciudadanos. Los medios nos proponen los temas ya jerarquizados, haciendo que unos sean más importantes o relevantes que otros, y alguno de ellos puede ser tan importante que determine de alguna forma especial a todos los demás (es el fenómeno que en inglés se llama priming o preparación: por ejemplo, las terribles bombas del 11 de marzo, que hicieron que las elecciones de 2004 se leyeran en clave terrorista, que es una clave política, no económica). Y nos proponen también los temas de preocupación no de una forma neutra o puramente referencial, sino interpretados desde marcos o encuadres de interpretación: es el fenómeno que en inglés se llama framing/frame: encuadramiento, encuadre, enmarque, enmarcado, marco. Se llama Agenda de Atributos al conjunto de atributos que se explicitan en ese proceso de encuadramiento de la noticia: atributos positivos, negativos o neutros. Atributos que se transfieren a las agendas ciudadanas y forman parte del conjunto de la agenda mediática transferida. Pero la agenda mediática y las agendas ciudadanas (pública, personal) no son las únicas agendas relevantes, porque la agenda política o Institucional, que es aquello que los políticos y sus partidos o las diversas Instituciones sociales nos proponen como temas a pensar, esa agenda, a la que vamos a llamar agenda política, es particularmente importante e, incluso, dominante. La agenda política marca fuertemente a la agenda mediática y, a su través, a la agenda pública y a la agenda personal. Cuando intentamos contestar a esta pregunta compleja 
"Si las agendas ciudadanas las marca la agenda mediática (tesis central, perfectamente corroborada, mediática?, cuando queremos contestar a esto, digo, todo nos lleva a la agenda política. Esta agenda política así como los acontecimientos de la realidad sobre los que la prensa debe informar ineludiblemente, al margen de su adscripción ideológica, son las dos cosas que, probablemente, marcan con más fuerza la agenda mediática y, por tanto, nuestras propias agendas ciudadanas, la agenda pública y la agenda personal.

Para citar este artículo:

Bouza, Fermín (20-06-2012). LAS METODOLOGÍAS DE AGENDA EN EL ESTUDIO DE LA OPINIÓN PÚBLICA COMO BASE DE LA COMUNICACIÓN POLÍTICA.

REDMARKA - CIECID - Unidad de Investigación en Marketing Aplicado-Universidad de A Coruña Año V, Número 8, V1, pp.41-54

ISSN $1852-2300$

URL del Documento : cienciared.com.ar/ra/doc.php? $n=1654$

URL de la Revista : cienciared.com.ar/ra/revista.php?wid=39 